PROCEEDINGS OF THE

AMERICAN MATHEMATICAL SOCIETY

Volume 137, Number 11, November 2009, Pages 3689-3693

S 0002-9939(09)09998-5

Article electronically published on June 11, 2009

\title{
ON THE AMENABILITY OF PARTIAL AND ENVELOPING ACTIONS
}

\author{
FERNANDO ABADIE AND LAURA MARTÍ PÉREZ
}

(Communicated by Marius Junge)

\begin{abstract}
We prove that a partial action is amenable if and only if so is its Morita enveloping action. As applications we prove that any partial representation of a discrete group is positive definite, and we extend a result of Zeller-Meier concerning the amenability of discrete groups and the existence of invariant states to partial actions.
\end{abstract}

In [1, the first-named author studied enveloping actions of partial actions on topological spaces and $C^{*}$-algebras and compared some of the structures naturally associated to actions, namely, crossed products. However, most of the results obtained in that work correspond to spatial constructions, that is, to reduced crossed products. The goal of the present paper is to give versions of some of the work in [1] for full crossed products, and then to combine both the spatial and the universal versions to the study of some amenability questions.

Before proceeding we recall next some of the necessary background on partial actions and their enveloping actions.

If $A$ is a $C^{*}$-algebra, a partial action of the group $G$ on $A$ is a pair $\alpha=$ $\left(\left\{D_{t}\right\}_{t \in G},\left\{\alpha_{t}\right\}_{t \in G}\right)$, where $D_{t}$ is a closed ideal of $A$ and $\alpha_{t}: D_{t^{-1}} \rightarrow D_{t}$ is an isomorphism subject to the conditions:

(1) $\alpha_{e}=I d_{A}$, thus $D_{e}=A$, where $e$ is the identity of $G$, and

(2) $\alpha_{s t}(a)=\alpha_{s}\left(\alpha_{t}(a)\right)$ whenever $a \in D_{t^{-1}}$ is such that $\alpha_{t}(a) \in D_{s^{-1}}$ (so that $\left.a \in D_{t^{-1} s^{-1}}\right)$.

For instance if $\beta: G \times B \rightarrow B$ is an action of $G$ on the $C^{*}$-algebra $B$ and $A \triangleleft B$, then the restriction $\left.\beta\right|_{A}$ of $\beta$ to $A$ is a partial action of $G$ on $A$. More precisely, $\left.\beta\right|_{A}=\left(\left\{D_{t}\right\},\left\{\alpha_{t}\right\}\right)$, where $D_{t}=A \cap \beta_{t}(A)$, and $\alpha_{t}(a)=\beta_{t}(a), \forall a \in D_{t^{-1}}, t \in G$. If $\alpha$ is a partial action on $A$ for which there exists such an action $\beta$ in a larger algebra $B$ containing $A$ as a closed ideal and in addition $B=\overline{\operatorname{span}}\left\{\beta_{t}(a): a \in A, t \in G\right\}$, then it is said that $\beta$ is an enveloping action of $\alpha$. The enveloping action is unique up to isomorphism, if it exists, which is not always the case. On the other hand, given a partial action $\alpha$, there always exists another partial action $\alpha^{\prime}$, which is Morita equivalent to $\alpha$, such that $\alpha^{\prime}$ does have an enveloping action $\beta^{\prime}$. For this reason $\beta^{\prime}$ is called a Morita enveloping action of $\alpha$. Thus every partial action has a Morita enveloping action that, in addition, is unique up to Morita equivalence of partial actions. Moreover the reduced crossed products of Morita equivalent partial

Received by the editors December 10, 2007.

2000 Mathematics Subject Classification. Primary 46L55.

(C)2009 American Mathematical Society Reverts to public domain 28 years from publication 
actions are Morita equivalent, as well as the reduced crossed products by a partial action and by its Morita enveloping action.

Suppose now that $\mathcal{B}$ is a Fell bundle over the locally compact group $G$. Then the Banach *-algebra $L^{1}(\mathcal{B})$ has associated two distinguished $C^{*}$-completions. One of them is $C^{*}(\mathcal{B}):=C^{*}\left(L^{1}(\mathcal{B})\right.$ ) (the enveloping $C^{*}$-algebra of $L^{1}(\mathcal{B})$ ), called the full cross-sectional $C^{*}$-algebra of $\mathcal{B}$, whose norm is the maximal $C^{*}$-norm on $L^{1}(\mathcal{B})$. The other one is $C_{r}^{*}(\mathcal{B}):=\overline{\Lambda\left(L^{1}(\mathcal{B})\right)} \subseteq \mathcal{L}\left(L^{2}(\mathcal{B})\right)$, the so-called reduced cross-sectional algebra of $\mathcal{B}$, where $\Lambda$ is the left regular representation of $L^{1}(\mathcal{B})$ on the full right $B_{e^{-}}$ Hilbert module $L^{2}(\mathcal{B})$, and $\mathcal{L}\left(L^{2}(\mathcal{B})\right)$ denotes as usual the $C^{*}$-algebra of adjointable operators on $L^{2}(\mathcal{B})$. The norm of $C_{r}^{*}(\mathcal{B})$ is called the reduced norm. The regular representation extends to $\Lambda: C^{*}(\mathcal{B}) \rightarrow C_{r}^{*}(\mathcal{B})$, also called regular, such that it is surjective. When this $\Lambda$ is an isomorphism, it is said that $\mathcal{B}$ is amenable. Finally, if $\alpha$ is a partial action on the $C^{*}$-algebra $A$, it has an associated Fell bundle $\mathcal{B}_{\alpha}$. Then $A \rtimes_{\alpha} G:=C^{*}\left(\mathcal{B}_{\alpha}\right)$ and $A \rtimes_{\alpha, r} G:=C_{r}^{*}\left(\mathcal{B}_{\alpha}\right)$ are called respectively the (full) crossed product and the reduced crossed product of $A$ by $\alpha$. The partial action is said to be amenable if $\mathcal{B}_{\alpha}$ is amenable. The reader is referred to [1, [3, , 4] and [5] for more details on Fell bundles and their amenability.

\section{Amenability of partial actions}

Recall from [1, Definition 4.2] that a right ideal $\mathcal{E}=\left(E_{t}\right)_{t \in G}$ of a Fell bundle $\mathcal{B}=\left(B_{t}\right)_{t \in G}$ is a sub-Banach bundle of $\mathcal{B}$ such that $\mathcal{E B} \subseteq \mathcal{E}$. The next theorem is the main result of the paper.

Theorem 1.1. Let $\mathcal{B}=\left(B_{t}\right)_{t \in G}$ be a Fell bundle over the locally compact group $G, \mathcal{A}=\left(A_{t}\right)_{t \in G}$ a sub-Fell bundle of $\mathcal{B}$, and $\mathcal{E}=\left(E_{t}\right)_{t \in G}$ a right ideal of $\mathcal{B}$ such that $\mathcal{A} \subseteq \mathcal{E}$ and $\operatorname{span}\left(\mathcal{E}^{*} \mathcal{E} \cap B_{t}\right)$ is dense in $B_{t}$, for all $t \in G$. Then the completion $C^{*}(\mathcal{E})$ of $L^{1}(\mathcal{E})$ in $C^{*}(\mathcal{B})$ is a bimodule implementing a Morita equivalence between $C^{*}(\mathcal{A})$ and $C^{*}(\mathcal{B})$. Moreover $\mathcal{A}$ is amenable if and only if $\mathcal{B}$ is amenable.

Proof. From [1, Theorem 3.2] we have that $L^{1}(\mathcal{A}) L^{1}(\mathcal{E}) \subseteq L^{1}(\mathcal{E}), L^{1}(\mathcal{E}) L^{1}(\mathcal{E})^{*}=$ $L^{1}(\mathcal{A})$, and that $L^{1}(\mathcal{E})$ is a right $L^{1}(\mathcal{B})$-module such that $\overline{\operatorname{span}} L^{1}(\mathcal{E})^{*} L^{1}(\mathcal{E})=$ $L^{1}(\mathcal{B})$. Therefore $L^{1}(\mathcal{E})$ is a $\left(L^{1}(\mathcal{A})-L^{1}(\mathcal{B})\right)$-bimodule. Let \|\|$_{\mathcal{A}}$ and \|\|$_{\mathcal{B}}$ be the maximal $C^{*}$-norms on $L^{1}(\mathcal{A})$ and $L^{1}(\mathcal{B})$ respectively. We clearly have that $\|f\|_{\mathcal{A}} \geq\|f\|_{\mathcal{B}}, \forall f \in L^{1}(\mathcal{A})$. For $\xi \in L^{1}(\mathcal{E})$ define $\|\xi\|_{E}^{2}:=\left\|\xi * \xi^{*}\right\|_{\mathcal{A}}$, and $\|\xi\|_{F}^{2}:=$ $\left\|\xi^{*} * \xi\right\|_{\mathcal{B}}$. Then $\|\xi\|_{F}^{2}=\left\|\xi^{*} * \xi\right\|_{\mathcal{B}}=\left\|\xi * \xi^{*}\right\|_{\mathcal{B}} \leq\left\|\xi * \xi^{*}\right\|_{\mathcal{A}}=\|\xi\|_{E}^{2}$. Also let $E$ and $F$ be the completions of $L^{1}(\mathcal{E})$ with respect to \|\|$_{E}$ and \|\|$_{F}$ respectively. Then the module structures of $L^{1}(\mathcal{E})$ extend to $E$ and $F$, so that $E$ is a left full $C^{*}(\mathcal{A})$-Hilbert module, and $F$ is a right full $C^{*}(\mathcal{B})$-Hilbert module, where $\langle\xi, \eta\rangle_{E}=\xi * \eta^{*}$ and $\langle\xi, \eta\rangle_{F}=\xi^{*} * \eta$ for $\xi, \eta \in L^{1}(\mathcal{E})$ (note that $F=C^{*}(\mathcal{E})$ ). To prove the first assertion of the theorem, by [8], it is enough to show that $\|\xi\|_{E}=\|\xi\|_{F}, \forall \xi \in L^{1}(\mathcal{E})$. To this purpose consider the set $J:=\operatorname{span}\left\{\xi^{*} * \eta: \xi, \eta \in L^{1}(\mathcal{E})\right\}$, which is a dense *-ideal of $L^{1}(\mathcal{B})$. Given $g \in L^{1}(\mathcal{B})$, denote by $C_{g}: L^{1}(\mathcal{E}) \rightarrow L^{1}(\mathcal{E})$ the convolution operator such that $C_{g}(\zeta)=\zeta * g, \forall \zeta \in \mathcal{E}$. Recall that if $x, y \in E$, then $\theta_{x, y}: E \rightarrow E$ is defined as $\theta_{x, y}(z)=\langle z, x\rangle_{E} y$, and the $C^{*}$-algebra $\mathcal{K}_{C^{*}(\mathcal{A})}$ of compact operators on the $C^{*}(\mathcal{A})$-Hilbert module $E$ is the completion of $\operatorname{span}\left\{\theta_{x, y}: x, y \in E\right\}$ with respect to the operator norm. Note now that if $\xi_{1}, \ldots, \xi_{n}, \eta_{1}, \ldots, \eta_{n} \in L^{1}(\mathcal{E})$ and $g=\sum_{j=1}^{n} \xi_{j}^{*} * \eta_{j} \in J$, then $C_{g}(\zeta)=\sum_{j=1}^{n}\left(\zeta * \xi_{j}^{*}\right) * \eta_{j}=\sum_{j=1}^{n} \theta_{\xi_{j}, \eta_{j}}(\zeta)$. That is, $C_{g}$ is precisely the restriction of $\sum_{j=1}^{n} \theta_{\xi_{j}, \eta_{j}}$ to $L^{1}(\mathcal{E})$, or, in other words, $C_{g}$ is \|\|$_{E}$-bounded, and its continuous extension to $E$ is precisely the compact operator 
$\sum_{j=1}^{n} \theta_{\xi_{j}, \eta_{j}}$. Let $\rho^{\prime}: J \rightarrow \mathcal{K}_{C^{*}(\mathcal{A})}$ be the map such that $\rho^{\prime}(g)$ is the continuous extension of $C_{g}$ to all of $E$. This is a homomorphism of *-algebras. Since $J$ is a *ideal of the Banach algebra $L^{1}(\mathcal{B})$ and $\mathcal{K}_{C^{*}(\mathcal{A})}$ is a $C^{*}$-algebra, then by [5. XI-19.11] $\rho^{\prime}$ extends uniquely to a homomorphism of Banach ${ }^{*}$-algebras $\rho: L^{1}(\mathcal{B}) \rightarrow \mathcal{K}_{C^{*}(\mathcal{A})}$. This implies that $\|\rho(g)\| \leq\|g\|_{\mathcal{B}}, \forall g \in L^{1}(\mathcal{B})$. On the other hand, for $\xi \in L^{1}(\mathcal{E})$ we have $\rho^{\prime}\left(\xi^{*} * \xi\right)=\theta_{\xi, \xi}$. Hence:

$$
\|\xi\|_{E}^{2}=\left\|\theta_{\xi, \xi}\right\|=\left\|\rho^{\prime}\left(\xi^{*} * \xi\right)\right\| \leq\left\|\xi^{*} * \xi\right\|_{\mathcal{B}}=\|\xi\|_{F}^{2} \leq\|\xi\|_{E}^{2},
$$

where the last inequality was shown after the definition of both norms. This shows that \|\|$_{E}=\|\|_{F}$, which ends the proof of the first part of the theorem.

By [1, Corollary 3.2] we have that the amenability of $\mathcal{A}$ implies that of $\mathcal{B}$. So to prove the second assertion of the theorem, suppose $\mathcal{B}$ is amenable. We have just seen that the maximal norm on $L^{1}(\mathcal{A})$ is the restriction to this algebra of the maximal norm of $L^{1}(\mathcal{B})$, a fact that was already known for the reduced norms ([1, Corollary 3.1]). So if the maximal and the reduced norms of $L^{1}(\mathcal{B})$ agree, their restrictions to $L^{1}(\mathcal{A})$ also coincide; that is, $\mathcal{A}$ is amenable whenever $\mathcal{B}$ is amenable.

Remark 1.2. A more precise result can be given: there are isomorphisms of partially ordered sets between the families of $C^{*}$-seminorms on $L^{1}(\mathcal{A}), L^{1}(\mathcal{E})$ and $L^{1}(\mathcal{B})$, such that for corresponding $C^{*}$-seminorms the respective completions form a Morita equivalence system. See [2 for details.

The theorem above allows us to adapt in a straightforward way the proofs of Theorem 3.3, Proposition 4.5 and Proposition 4.6 of [1] to the case of full crossed products, an easy task that we leave to the reader. Then the following corollaries are obtained:

Corollary 1.3. If the partial action $\alpha$ of the locally compact group $G$ on the $C^{*}$ algebra $A$ has a Morita enveloping action $\beta$ acting on the $C^{*}$-algebra $B$, then $A \rtimes_{\alpha} G$ and $B \rtimes_{\beta} G$ are Morita equivalent. Moreover $\alpha$ is amenable if and only if $\beta$ is amenable.

Corollary 1.4. If $\alpha_{1}$ and $\alpha_{2}$ are Morita equivalent partial actions, then $A \rtimes_{\alpha_{1}} G$ and $B \rtimes_{\alpha_{2}} G$ are Morita equivalent. Moreover $\alpha_{1}$ is amenable if and only if $\alpha_{2}$ is amenable.

\section{INVARIANT STATES}

Let $\alpha$ be a partial action of a group $G$ on a $C^{*}$-algebra $A$. A map $\phi$ on $A$ is called $\alpha$-invariant whenever $\phi\left(\alpha_{t}(a)\right)=\phi(a), \forall t \in G$ and $a \in D_{t^{-1}}$. We will denote by $A_{\alpha}^{\prime}$ the Banach space of $\alpha$-invariant bounded linear functionals on $A$. The space $A_{\alpha}^{\prime}$ is partially ordered: if $\phi_{1}, \phi_{2} \in A_{\alpha}^{\prime}$, then $\phi_{1} \geq \phi_{2}$ if $\phi_{1}-\phi_{2}$ is a positive linear functional.

In this section we show that if the partial action $\alpha$ on the $C^{*}$-algebra $A$ has enveloping action $\beta$, acting on a unital $C^{*}$-algebra $B$, then the map $\psi \mapsto \frac{\left.\psi\right|_{A}}{\left\|\left.\psi\right|_{A}\right\|}$ is a bijection between the set of $\beta$-invariant states of $B$ onto the set of $\alpha$-invariant states of $A$. The requirement that $B$ is unital is necessary.

Proposition 2.1. Let $\alpha=\left(\left\{D_{t}\right\},\left\{\alpha_{t}\right\}\right)$ be a partial action of the locally compact group $G$ on the $C^{*}$-algebra $A$, and suppose that $\alpha$ has an enveloping action $\beta$ acting on a $C^{*}$-algebra $B$. Then the map $R_{A}: B_{\beta}^{\prime} \rightarrow A_{\alpha}^{\prime}$ given by $\left.\psi \mapsto \psi\right|_{A}$ is an 
injective bounded linear map that preserves the order. If $B$ is unital, then $R_{A}$ is an isomorphism of Banach spaces and ordered sets. Therefore, if $B$ is unital, there exists a $\beta$-invariant state of $B$ if and only if there exists an $\alpha$-invariant state of $A$.

Proof. It is clear that $\left.\psi \mapsto \psi\right|_{A}$ is linear and contractive. Suppose that $\psi \in B_{\beta}^{\prime}$, and let $\phi=\left.\psi\right|_{A}$. Then $\phi$ is a nonzero functional: otherwise we would have, for every $b=\sum_{j=1}^{n} \beta_{t_{j}}\left(a_{j}\right), a_{j} \in A \forall j: \psi(b)=\sum_{j=1}^{n} \psi\left(\beta_{t_{j}}\left(a_{j}\right)\right)=\sum_{j=1}^{n} \psi\left(a_{j}\right)=$ $\sum_{j=1}^{n} \phi\left(a_{j}\right)=0$, which implies $\psi=0$. The functional $\phi$ is $\alpha$-invariant: if $a \in D_{t^{-1}}$, then $\phi\left(\alpha_{t}(a)\right)=\psi\left(\beta_{t}(a)\right)=\psi(a)=\phi(a)$. It is clear that $\phi$ is positive if $\psi$ is positive. This shows that $R_{A}: B_{\beta}^{\prime} \rightarrow A_{\alpha}^{\prime}$ is an injective contractive linear map that preserves the order.

Suppose conversely that $\phi$ is an $\alpha$-invariant bounded linear functional on $A$, and that $B$ is unital. Then $\phi_{t}:=\phi \beta_{t}^{-1}$ is a bounded linear functional on $\beta_{t}(A)$. Since $B$ is unital and the sum of the ideals $\beta_{t}(A)$ is a dense ideal in $B$, there exist elements $t_{1}, \ldots, t_{k}$ in $G$ such that $B=A_{1}+\cdots+A_{k}$, where $A_{j}=\beta_{t_{j}}(A)$, $\forall j=1, \ldots, k$. Let $u_{j} \in A_{j}$ be such that $1=u_{1}+\cdots+u_{k}$, and let $v_{j}=$ $\beta_{t_{j}}^{-1}\left(u_{j}\right) \in A, \forall j=1, \ldots, k$. Define $\psi: B \rightarrow \mathbb{C}$ by $\psi(b)=\sum_{j=1}^{k} \phi_{t_{j}}\left(b u_{j}\right)$, $\forall b \in B$, which is clearly a bounded linear functional on $B$. Note that if $a \in A$ we have $a u_{j} \in A \cap \beta_{t_{j}}(A)=D_{t_{j}}$. Since $\phi$ is $\alpha$-invariant, it follows that $\psi(a)=$ $\sum_{j=1}^{k} \phi_{t_{j}}\left(a u_{j}\right)=\sum_{j=1}^{k} \phi \beta_{t_{j}^{-1}}\left(a u_{j}\right)=\sum_{j=1}^{k} \phi\left(\alpha_{t_{j}^{-1}}\left(a u_{j}\right)\right)=\phi\left(\sum_{j=1}^{k} a u_{j}\right)=\phi(a)$. Therefore $\left.\psi\right|_{A}=\phi$. Now for $a \in A$ and $t \in G$, we have $\beta_{t}(a) u_{j} \in A_{j}$ and $\phi_{j}\left(\beta_{t}(a) u_{j}\right)=\phi\left(\beta_{t_{j}^{-1} t}(a) v_{j}\right)$. Since $\beta_{t_{j}^{-1} t}(a) v_{j} \in D_{t_{j}^{-1} t}$ we have $\phi_{j}\left(\beta_{t}(a) u_{j}\right)=$

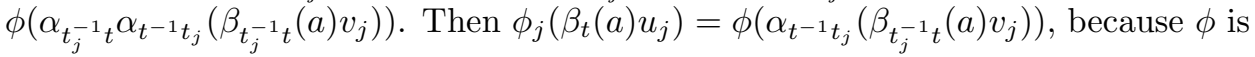
$\alpha$-invariant. Thus $\phi_{j}\left(\beta_{t}(a) u_{j}\right)=\phi\left(\alpha_{t^{-1} t_{j}}\left(\beta_{t_{j}^{-1} t}(a) v_{j}\right)\right)=\phi\left(\beta_{t^{-1} t_{j}}\left(\beta_{t_{j}^{-1} t}(a) v_{j}\right)\right)=$ $\phi\left(a \beta_{t^{-1}}\left(u_{j}\right)\right)$. Hence, since $\sum_{j=1}^{k} \beta_{t^{-1}}\left(u_{j}\right)=1$ we get

$$
\psi\left(\beta_{t}(a)\right)=\sum_{j=1}^{k} \phi_{j}\left(\beta_{t}(a) u_{j}\right)=\phi\left(a \sum_{j=1}^{k} \beta_{t^{-1}}\left(u_{j}\right)\right)=\phi(a)=\psi(a) .
$$

The $\beta$-invariance of $\psi$ follows now by its linearity. This shows that $R_{A}$ is also surjective, thus an isomorphism of Banach spaces. Moreover if $b \in B^{+}$, then $b=\beta_{t_{1}}\left(a_{1}\right)+\cdots+\beta_{t_{k}}\left(a_{k}\right)$, with $a_{j} \in A^{+}$, from which we have that $\psi(b)=$ $\sum_{j=1}^{k} \psi\left(\beta_{t_{j}}\left(a_{j}\right)\right)=\sum_{j=1}^{k} \phi\left(a_{j}\right) \geq 0$. Therefore $R_{A}$ is also an isomorphism of partially ordered sets. Finally, from the above, it follows that $\psi \mapsto \frac{\psi}{\|\psi\|}$ is a bijection from the set of $\beta$-invariant states of $B$ onto the set of $\alpha$-invariant states of $A$.

When $B$ is not unital, it is not true that every positive $\alpha$-invariant linear functional on $A$ can be extended to a $\beta$-invariant linear functional on $B$. For instance, consider $B=C_{0}(\mathbb{R})$ and $\beta: \mathbb{R} \times B \rightarrow B$ such that $\beta_{x}(b)(t):=b(t-x)$. Let $A=C_{0}(0,1)$ and $\alpha:=\left.\beta\right|_{A}$. It is clear that $\beta$ is the enveloping action of $\alpha$. Now the functional $\phi: A \rightarrow \mathbb{C}$ such that $\phi(a)=\int_{[0,1]} a d m$, where $m$ is Lebesgue measure, is $\alpha$-invariant. On the other hand, Lebesgue measure on $\mathbb{R}$ is translation invariant, so if $\phi$ could be extended to a $\beta$-invariant linear functional on $B$, this functional on the dense subalgebra $C_{c}(\mathbb{R})$ should be integration with respect to Lebesgue measure, which is not a bounded linear map. However, as shown in [6], in the commutative case one can see that any probability measure on the spectrum $\hat{A}$ of $A$ can be uniquely extended to a Radon measure on the spectrum $\hat{B}$ of $B$. 


\section{PARTial aCtions of Discrete Groups}

In this final small section we give two applications of the preceding results.

Theorem 3.1. Let $u: G \rightarrow B(H)$ be a partial representation of a discrete group $G$. Then $u$ is a positive definite map.

Proof. Just observe that in view of Corollary 1.3 above it is possible now to remove the hypothesis of amenability of the group $G$ that was necessary in 1 , Proposition 3.3].

A Fell bundle over an amenable group $G$ is necessarily amenable ([3, 4]). As a partial converse of this fact, for Fell bundles associated to partial actions of discrete groups we have the following result:

Theorem 3.2. Let $\alpha$ be a partial action of the discrete group $G$ on the $C^{*}$-algebra $A$, and suppose that $\alpha$ has enveloping action $\beta$ acting on a unital $C^{*}$-algebra $B$. Then the following are equivalent:

(i) $G$ is amenable.

(ii) $\beta$ is amenable and there exists a $\beta$-invariant state in $B$.

(iii) $\alpha$ is amenable and there exists an $\alpha$-invariant state in $A$.

Proof. The equivalence between the first two assertions is [7, 5.2]. Combining Corollary 1.3 and Proposition 2.1 we obtain the equivalence between the last two assertions.

\section{REFERENCES}

[1] Fernando Abadie, Enveloping actions and Takai duality for partial actions, J. Funct. Anal. 197 (2003), 14-67. MR1957674 (2004c:46130)

[2] Fernando Abadie, Applications of ternary rings to $C^{*}$-algebras and locally $C^{*}$-algebras, preprint, 2007.

[3] Ruy Exel, Amenability for Fell bundles, J. Reine Angew. Math. 492 (1997), 41-73. MR1488064 (99a:46131)

[4] Ruy Exel, Chi-Keung Ng, Approximation property of $C^{*}$-algebraic bundles, Math. Proc. Cambridge Philos. Soc. 132 (2002), no. 3, 509-522. MR1891686 (2002k:46189)

[5] J. M. G. Fell, R. S. Doran, Representations of *-algebras, locally compact groups, and Banach *-algebraic bundles, Pure and Applied Mathematics, vol. 126, Academic Press, Boston, MA, 1988. MR0936629 (90c:46002)

[6] Laura Martí, Condiciones de promediabilidad en fibrados de Fell, Master's Thesis, Universidad de la República, Montevideo, Uruguay, 2006.

[7] G. Zeller-Meier, Produits croisés d'une $C^{*}$-algèbre par un groupe d'automorphismes, J. Math. Pures et Appl., IX Sér., 47 (1968), 101-239. MR0241994 (39:3329)

[8] H. H. Zettl, A characterization of ternary rings of operators, Advances in Math. 48 (1983), 117-143. MR700979 (84h:46093)

Centro de Matemática, Facultad de Ciencias, Universidad de la República, Iguá 4225, 11400, Montevideo, Uruguay

E-mail address: fabadie@cmat.edu.uy

Centro de Matemática, Facultad de Ciencias, Universidad de la República, Iguá 4225, 11400, Montevideo, Uruguay - and - Department of Pure Mathematics, University of Waterloo, Waterloo, Ontario, Canada, N2L 3G1

E-mail address: lau@cmat.edu.uy

E-mail address: lrmarti@uwaterloo.ca 